183

\section{PREGNANCY IN ADOLESCENCE: ALWAYS UNWANTED?}

\author{
C. Meneses ${ }^{1}$, C.D.S. Lopes ${ }^{2}$ \\ ${ }^{1}$ NESA, ${ }^{2} I M S$, UERJ, Rio de Janeiro, Brazil
}

Introduction: Studies conducted all over the world show that the rates of pregnancy in adolescence are increasing especially in the early adolescence (10 to 14 years of age). Although the majority of pregnancies in this period are not desired some studies suggest that an expressive number of them could be even wanted.

\section{Aims:}

1) Assess the prevalence of pregnancy willingness and possible associations with socioeconomic and demographic factors and social support network in a population of pregnant adolescents

2) Verify if age should be an effect modifier in these associations.

Methods: Cross sectional study conducted with pregnant adolescents regularly attending two public maternity. A sample of 232 pregnant adolescents between 13 and 20 years of age (mean: 17.3) completed a self-reported questionnaire to access socioeconomic and demographic data.

Results: Prevalence of pregnancy willingness was $46.2 \%$. Final adjusted model showed that being married (PR = 1.80; Cl 95\% 1.27-2.56) and do not have friends ( $P R=1.48 ; \mathrm{Cl} 95 \% 1.15-1.90)$ showed association with desire of being pregnant. Girls studying in the elementary grade and with 12 to 16 years of age showed less desire of being pregnant $(P R=0.57$; IC 95\% 0.38-0.88).

Conclusion: Pregnancy may not always be unwanted. Some factors, as being married and do not have friends may have influence in these cases. Professionals dealing with adolescents should be aware of these issues to identify risk situations that could be successfully managed.
184

\section{A SCORE FOR ASSESSING THE RISK OF ADOLESCENT PREGNANCY}

\author{
M.I. Santos ${ }^{1}$, F. Rosário² \\ ${ }^{1}$ Pediatrics, Hospital de São Teotónio, Viseu, \\ ${ }^{2}$ Primary Care, Centro de Saúde do Barreiro, \\ Barreiro, Portugal
}

Background: Adolescent pregnancy is an important health issue. Preventive programs usually focus on repeated pregnancy prevention, ignoring adolescents at high risk of first time pregnancy.

Aims: To analyze if socio-economic status, family type and adolescents' mothers' age at their first pregnancy behave as risk factors and to design a score to assess the risk for first time adolescent pregnancy.

Methods: A case-control study was performed: cases were primiparous adolescents, controls were primiparous adult women. Participants were asked about family structure and socio-economic status during adolescence and their mother's age at first pregnancy. Odds Ratio (OR) and respective 95\% confidence intervals $(\mathrm{Cl})$ were calculated. A first time adolescent pregnancy risk score was designed based on a multiple logistic regression model.

Results: A total of 66 adolescent and 140 adults were interviewed. Belonging to a family other than nuclear or extended types was related to high probability of getting pregnant during adolescence (OR: 9.37; Cl: 3.36-38.07). Low socio-economic status and having an adolescent mother didn't behave as risk factors when isolated (OR: 1.04; Cl: 0.34-5.11 and OR: 1.13; Cl: 0.42-4.32, respectively). They were only significant when simultaneously present (OR: 6.01; Cl: 2.36-20.79). The presence of all these factors determined the highest adolescent pregnancy probability (OR: 10.49; Cl: 3.43-51.00). A first time adolescent pregnancy risk assessment score was created, with an estimated sensitivity of $75.8 \%$ and specificity of $75.0 \%$.

Conclusions: Adolescent pregnancy is associated with unfavourable precedents that can be used to identify adolescents at increased risk of early pregnancy. 